\title{
PERIODIC SOLUTIONS AND STABILITY FOR A DELAYED DISCRETE RATIO-DEPENDENT PREDATOR-PREY SYSTEM WITH HOLLING-TYPE FUNCTIONAL RESPONSE
}

\author{
LIN-LIN WANG AND WAN-TONG LI
}

Received 1 October 2003

The existence of positive periodic solutions for a delayed discrete predator-prey model with Holling-type-III functional response $N_{1}(k+1)=N_{1}(k) \exp \left\{b_{1}(k)-a_{1}(k) N_{1}\left(k-\left[\tau_{1}\right]\right)\right.$ $\left.-\alpha_{1}(k) N_{1}(k) N_{2}(k) /\left(N_{1}^{2}(k)+m^{2} N_{2}^{2}(k)\right)\right\}, N_{2}(k+1)=N_{2}(k) \exp \left\{-b_{2}(k)+\alpha_{2}(k) N_{1}^{2}(k-\right.$ $\left.\left.\left[\tau_{2}\right]\right) /\left(N_{1}^{2}\left(k-\left[\tau_{2}\right]\right)+m^{2} N_{2}^{2}\left(k-\left[\tau_{2}\right]\right)\right)\right\}$ is established by using the coincidence degree theory. We also present sufficient conditions for the globally asymptotical stability of this system when all the delays are zero. Our investigation gives an affirmative exemplum for the claim that the ratio-dependent predator-prey theory is more reasonable than the traditional prey-dependent predator-prey theory.

\section{Introduction}

The current spectacular interest in ecological systems has built up gradually; it can be traced back to the years after the First World War. Since the pioneering theoretical works by Lotka and Volterra early at that time, the study of realistic mathematical models in ecology, especially the study of the relations between species and their environment, has become a very popular topic that interested mathematicians as well as biologists. Investigations on various population models reflect their use in helping to understand the dynamic processes involved in such areas as predator-prey and competition, renewable resource management, evolution of pesticide resistant strains, ecological control of pests, multispecies societies, plant-herbivore systems, and so on. The increasing use of mathematics in biology is inevitable as biology becomes more quantitative. In fact, most realistic biology problems could be solved on the fundament of constructing suitable mathematical models. In many such models, the key terms specifying the outcome of predator-prey interactions are the functional and numerical responses, which reflect the relationship between predators and their prey. The dynamic relationship between predators and their prey has long been and will continue to be one of the dominant themes in both ecology and mathematical ecology due to its universal existence and importance (see Berryman [4]). Although these problems may appear to be mathematically simple at first sight, they are, in fact, very challenging and complicated. There are many different kinds of 
predator-prey models in the literature; for more details, we refer to $[4,8]$. In general, a predator-prey system takes the form

$$
\begin{aligned}
& x^{\prime}=r x\left(1-\frac{x}{K}\right)-\varphi(x) y, \\
& y^{\prime}=y(\mu \varphi(x)-D)
\end{aligned}
$$

where $\varphi(x)$ is the functional response function, which reflects the capture ability of the predator. For more biological meaning, the reader may consult $[8,24]$. Massive work has been done on this issue. We refer to the monographs $[10,19,25,28]$ for general delayed biological systems and to [13,27, 29, 30, 31, 33, 35] for investigations on predator-prey systems. Here $\varphi(x)$ may be in different forms: the standard Holling-type-I, type-II, and type-III response functions [12], Ivlev's functional response [17], and Rosenzweig's functional response [26]. For the most examples that appear in the literature, it is assumed that $\varphi(x)$ has the second continuous derivative and satisfies the following assumptions: (i) $\varphi(0)=0$; (ii) $\varphi^{\prime}(x)>0$ for $x \geq 0$; (iii) $\varphi^{\prime \prime}(x)<0$ for $x>0$; (iv) $\lim _{x \rightarrow+\infty} \varphi(x)=c<\infty$.

Recently, there is growing explicit biological and physiological evidence $[3,13,18,20]$ that in many situations, especially when predators have to search for food (and, therefore, have to share or compete for food), a more suitable general predator-prey theory should be based on the so-called ratio-dependent theory, which can be roughly stated as that the per capita predator growth rate should be a function of the ratio of prey-to-predator abundance, and so should be the so-called ratio-dependent functional response (thus the traditional model (1.1) should be named as prey-dependent model [14]). This is strongly supported by numerous field and laboratory experiments and observations [2, 11]. A general form of a ratio-dependent model is

$$
\begin{aligned}
& x^{\prime}=r x\left(1-\frac{x}{K}\right)-\varphi\left(\frac{x}{y}\right) y, \\
& y^{\prime}=y\left(\mu \varphi\left(\frac{x}{y}\right)-D\right) .
\end{aligned}
$$

Here the predator-prey interactions are described by $\varphi(x / y)$ instead of $\varphi(x)$ in (1.1). This can be interpreted as follows. When the numbers of predators change slowly (relative to the change of their prey), there is often competition among the predators, and the per capita rate of predation depends on the numbers of both preys and predators, most likely and simply on their ratio. For system (1.2) with periodic coefficients, Fan et al. [7] explored the existence of periodic solutions with delays. In addition, most research papers concentrate on the so-called Michaelis-Menten-type ratio-dependent predatorprey model

$$
\begin{aligned}
& x^{\prime}=r x\left(1-\frac{x}{K}\right)-\frac{\alpha x y}{m y+x} \\
& y^{\prime}=y\left(-d+\frac{f x}{m y+x}\right)
\end{aligned}
$$


see $[3,13,18,20,32]$ and the references therein. In view of periodicity of the actual environment, Fan and Wang [5] established verifiable criteria for the global existence of positive periodic solutions of a more general delayed ratio-dependent predator-prey model with periodic coefficients of the form

$$
\begin{aligned}
& x^{\prime}(t)=x(t)\left[a(t)-b(t) \int_{-\infty}^{t} k(t-s) x(s) d s\right]-\frac{c(t) x(t) y(t)}{m y(t)+x(t)} \\
& y^{\prime}(t)=y(t)\left[\frac{f(t) x(t-\tau(t))}{m y(t-\tau)+x(t-\tau)}-d(t)\right] .
\end{aligned}
$$

The functional response function $\varphi(u)=c u /(m+u), u=x / y$, in above models was used by Holling as Holling-type-II function; they usually describe the uptake of substrate by the microorganisms in microbial dynamics or chemical kinetics [8].

But when we describe the relationship between higher animals, a more suitable response function,

$$
\varphi(x)=\frac{\mu x^{2}}{1+\rho x^{2}},
$$

should be taken into consideration in the predator-prey interactions, which is proposed by Holling [12] based on experiment. This response function is usually called the Hollingtype-III response function [8]. In our previous papers [29, 30], we established verifiable criteria for the global existence of positive periodic solutions and the stability of the following delayed predator-prey model with Holling-type-III response function:

$$
\begin{aligned}
& N_{1}^{\prime}(t)=N_{1}(t)\left[b_{1}(t)-a_{1}(t) N_{1}\left(t-\tau_{1}(t)\right)-\frac{\alpha_{1}(t) N_{1}(t)}{1+m N_{1}^{2}(t)} N_{2}(t-\sigma(t))\right], \\
& N_{2}^{\prime}(t)=N_{2}(t)\left[-b_{2}(t)-a_{2}(t) N_{2}(t)+\frac{\alpha_{2}(t) N_{1}^{2}\left(t-\tau_{2}(t)\right)}{1+m N_{1}^{2}\left(t-\tau_{2}(t)\right)}\right]
\end{aligned}
$$

where $N_{1}(t), N_{2}(t)$ are the densities of the prey population and predator population at time $t, b_{i}: \mathbb{R} \rightarrow \mathbb{R}, a_{i}, \tau_{i}, \sigma, \alpha_{i}: \mathbb{R} \rightarrow[0,+\infty)(i=1,2)$ are continuous functions of period $T$ and $\int_{0}^{T} b_{i}(t) d t>0, \alpha_{i}(t) \neq 0$, and $m$ is a nonnegative constant. And, in [31], we investigated the global existence of positive periodic solutions and the permanent property of the ratio-dependent predator-prey system with Holling-type-III functional response which takes the form

$$
\begin{aligned}
& x^{\prime}(t)=x(t)\left[a(t)-b(t) \int_{-\infty}^{t} k(t-s) x(s) d s\right]-\frac{c(t) x^{2}(t) y(t)}{m^{2} y^{2}(t)+x^{2}(t)}, \\
& y^{\prime}(t)=y(t)\left[\frac{e(t) x^{2}(t-\tau)}{m^{2} y^{2}(t-\tau)+x^{2}(t-\tau)}-d(t)\right]
\end{aligned}
$$

where the functional response function $\varphi(u)=c u^{2} /\left(1+m^{2} u^{2}\right), u=x / y ; a(t), b(t), c(t)$, $e(t)$, and $d(t)$ are all positive periodic continuous functions; and $m>0, \tau \geq 0$ are real constants.

On the other hand, though most predator-prey theories are based on continuous models governed by differential equations, the discrete-time models are more appropriate 
than the continuous ones when the size of the population is rarely small or the population has non-overlapping generations $[1,25]$. For the discrete Michaelis-Menten-type ratio-dependent predator-prey model, Fan and Wang [6] established the existence of positive periodic solutions.

Based on the above considerations, we pay our attention to the discrete-time analogue of the ratio-dependent predator-prey system with Holling-type-III functional response. We organize the present paper as follows. In the next section, by using the coincidence degree theory developed by Gaines and Mawhin [9], we will establish some sufficient conditions for the existence of at least one positive $\omega$-periodic solution. For the work concerning the existence of periodic solutions of delay differential equations which was done by using coincidence degree theory, we refer to $[15,16,21,22,23,31,34]$ and references cited therein. In view of the importance of the problem concerning the survival of species in ecological systems, we will present sufficient conditions for the globally asymptotical stability of the system in consideration when all the delays are zero in Section 3. A brief discussion is carried out in the final section.

\section{Existence of positive periodic solutions}

Throughout this paper, we always denote $\mathbb{Z}, \mathbb{Z}^{+}, \mathbb{R}, \mathbb{R}^{+}$, and $\mathbb{R}^{2}$ as the sets of all integers, nonnegative integers, real numbers, nonnegative real numbers, and two-dimensional Euclidean vector space, respectively.

We begin with the corresponding continuous ratio-dependent predator-prey system with Holling-type-III functional response:

$$
\begin{aligned}
& \frac{d N_{1}(t)}{d t}=N_{1}(t)\left[b_{1}(t)-a_{1}(t) N_{1}\left(t-\tau_{1}\right)\right]-\frac{\alpha_{1}(t) N_{1}^{2}(t) N_{2}(t)}{N_{1}^{2}(t)+m^{2} N_{2}^{2}(t)}, \\
& \frac{d N_{2}(t)}{d t}=N_{2}(t)\left[-b_{2}(t)+\frac{\alpha_{2}(t) N_{1}^{2}\left(t-\tau_{2}\right)}{N_{1}^{2}\left(t-\tau_{2}\right)+m^{2} N_{2}^{2}\left(t-\tau_{2}\right)}\right],
\end{aligned}
$$

where $N_{1}(t)$ and $N_{2}(t)$ represent the densities of the prey population and predator population, respectively, at time $t ; m>0, \tau_{1} \geq 0, \tau_{2} \geq 0$ are real constants; $b_{i}: \mathbb{R} \rightarrow \mathbb{R}$ and $a_{1}, \alpha_{i}$ : $\mathbb{R} \rightarrow \mathbb{R}^{+}(i=1,2)$ are continuous periodic functions with period $\omega>0$ and $\int_{0}^{\omega} b_{i}(t) d t>0$ $(i=1,2) ; b_{1}(t)$ stands for prey intrinsic growth rate; $b_{2}(t)$ stands for the death rate of the predator; $\alpha_{1}(t)$ and $\alpha_{2}(t)$ stand for the conversion rates; $m$ stands for half capturing saturation; the function $N_{1}(t)\left[b_{1}(t)-a_{1}(t) N_{1}\left(t-\tau_{1}\right)\right]$ represents the specific growth rate of the prey in the absence of the predator; and $N_{1}^{2}(t) /\left(N_{1}^{2}(t)+m^{2} N_{2}^{2}(t)\right)$ denotes the ratio-dependent response function, which reflects the capture ability of the predator.

We assume that the average growth rates in (2.1) change at regular intervals of time, then we can incorporate this aspect in (2.1) and obtain the following modified system:

$$
\begin{aligned}
& \frac{1}{N_{1}(t)} \frac{d N_{1}(t)}{d t}=b_{1}([t])-a_{1}([t]) N_{1}\left([t]-\left[\tau_{1}\right]\right)-\frac{\alpha_{1}([t]) N_{1}([t]) N_{2}([t])}{N_{1}^{2}([t])+m^{2} N_{2}^{2}([t])}, \\
& \frac{1}{N_{2}(t)} \frac{d N_{2}(t)}{d t}=-b_{2}([t])+\frac{\alpha_{2}([t]) N_{1}^{2}\left([t]-\left[\tau_{2}\right]\right)}{N_{1}^{2}\left([t]-\left[\tau_{2}\right]\right)+m^{2} N_{2}^{2}\left([t]-\left[\tau_{2}\right]\right)}, \quad t \neq 0,1,2, \ldots,
\end{aligned}
$$


where $[t]$ denotes the integer part of $t, t \in(0,+\infty)$. By a solution of $(2.2)$, we mean a function $N=\left(N_{1}, N_{2}\right)^{T}$, which is defined for $t \in(0,+\infty)$ and possesses the following properties:

(1) $N$ is continuous on $[0,+\infty)$;

(2) the derivatives $d N_{1}(t) / d t, d N_{2}(t) / d t$ exist at each point $t \in[0,+\infty)$ with the possible exception of the points $t \in\{0,1,2, \ldots\}$, where left-sided derivatives exist;

(3) the equations in (2.2) are satisfied on each interval $[k, k+1)$ with $k=0,1,2, \ldots$

On any interval of the form $[k, k+1), k=0,1,2, \ldots$, we can integrate (2.2) and obtain, for $k \leq t<k+1, k=0,1,2, \ldots$,

$$
\begin{aligned}
& N_{1}(t)=N_{1}(k) \exp \left\{\left[b_{1}(k)-a_{1}(k) N_{1}\left(k-\left[\tau_{1}\right]\right)-\frac{\alpha_{1}(k) N_{1}(k) N_{2}(k)}{N_{1}^{2}(k)+m^{2} N_{2}^{2}(k)}\right](t-k)\right\}, \\
& N_{2}(t)=N_{2}(k) \exp \left\{\left[-b_{2}(k)+\frac{\alpha_{2}(k) N_{1}^{2}\left(k-\left[\tau_{2}\right]\right)}{N_{1}^{2}\left(k-\left[\tau_{2}\right]\right)+m^{2} N_{2}^{2}\left(k-\left[\tau_{2}\right]\right)}\right](t-k)\right\} .
\end{aligned}
$$

Letting $t \rightarrow k+1$, we obtain from (2.3) that

$$
\begin{aligned}
& N_{1}(k+1)=N_{1}(k) \exp \left\{b_{1}(k)-a_{1}(k) N_{1}\left(k-\left[\tau_{1}\right]\right)-\frac{\alpha_{1}(k) N_{1}(k) N_{2}(k)}{N_{1}^{2}(k)+m^{2} N_{2}^{2}(k)}\right\}, \\
& N_{2}(k+1)=N_{2}(k) \exp \left\{-b_{2}(k)+\frac{\alpha_{2}(k) N_{1}^{2}\left(k-\left[\tau_{2}\right]\right)}{N_{1}^{2}\left(k-\left[\tau_{2}\right]\right)+m^{2} N_{2}^{2}\left(k-\left[\tau_{2}\right]\right)}\right\},
\end{aligned}
$$

which is a discrete-time analogue of system (2.1), where $N_{1}(t), N_{2}(t)$ are the densities of the prey population and predator population, respectively, at time $t$.

For convenience, in the sequel, we denote

$$
I_{\omega}=\{0,1, \ldots, \omega-1\}, \quad \bar{g}=\frac{1}{\omega} \sum_{k=0}^{\omega-1} g(k), \quad \bar{G}=\frac{1}{\omega} \sum_{k=0}^{\omega-1}|g(k)|,
$$

where $\{g(k)\}$ is an $\omega$-periodic sequence of real numbers defined for $k \in \mathbb{Z}$.

The exponential form of (2.4) assures that, for any initial condition $N(0)>0, N(k)$ remains positive. In the remainder of this paper, for biological reasons, we only consider solutions $N(k)$ with

$$
N_{i}(-k) \geq 0, \quad k=1,2, \ldots, \max \left\{\left[\tau_{1}\right],\left[\tau_{2}\right]\right\}, N_{i}(0)>0, i=1,2 .
$$

In order to obtain the existence of a positive periodic solution of system (2.4), we first make the following preparations.

Let $\Omega \subset \mathbb{R}^{n}$ be an open bounded set with closure $\bar{\Omega}$ and $f \in C^{1}\left(\Omega, \mathbb{R}^{n}\right) \cap C\left(\bar{\Omega}, \mathbb{R}^{n}\right)$. For $x \in \Omega$, let $J_{f}(x)$ denote the Jacobian determinant of $f$ at $x$ and let $S_{f}$ be the set of all critical points of $f$, that is, $S_{f}=\left\{x \in \Omega: J_{f}(x)=0\right\}$.

For $y \in \mathbb{R}^{n} \backslash f\left(\partial \Omega \cup S_{f}\right)$, that is, $y$ is a regular point of $f$, the degree of $f$ at $y$ is defined as

$$
\operatorname{deg}\{f, \Omega, y\}=\sum_{x \in f^{-1}(y)} \operatorname{sgn} J_{f}(x) .
$$


Let $X$ and $Z$ be two Banach spaces, Dom $L \subset X$ a subspace, and $L: \operatorname{Dom} L \rightarrow Z$ a linear mapping. The Kernel of $L$ is defined by $\operatorname{Ker} L=L^{-1}(0)$ and its $\operatorname{range}$ by $\operatorname{Im} L=L(\operatorname{Dom} L)$.

Let Coker $L=Z / \operatorname{Im} L$ be the quotient space of $Z$ under the equivalence relation $z^{\sim} z^{\prime} \Leftrightarrow$ $z-z^{\prime} \in \operatorname{Im} L$. Thus, Coker $L=\{z+\operatorname{Im} L: z \in Z\}$. So $\operatorname{dim} \operatorname{Coker} L=\operatorname{codim} \operatorname{Im} L$.

The linear mapping $L$ is called a Fredholm mapping if (i) $\operatorname{Im} L$ is closed in $Z$ and (ii) $\operatorname{Ker} L$ and Coker $L$ are finitely dimensional. When $L$ is a Fredholm mapping, its index is defined by $\operatorname{Ind} L=\operatorname{dim} \operatorname{Ker} L-\operatorname{codim} \operatorname{Im} L$. If $\operatorname{Ind} L=0$, then $L$ is called a Fredholm mapping of index zero.

If $L$ is a Fredholm mapping of index zero, then there exist continuous projections $P: X \rightarrow X$ and $Q: Z \rightarrow Z$ such that

$$
\operatorname{Im} P=\operatorname{Ker} L, \quad \operatorname{Im} L=\operatorname{Ker} Q=\operatorname{Im}(I-Q) .
$$

Define $L_{P}: \operatorname{Dom} L \cap \operatorname{Ker} P \rightarrow \operatorname{Im} L$ as the restriction $L_{\operatorname{Dom} L \cap \operatorname{Ker} P}$ of $L$ to $\operatorname{Dom} L \cap \operatorname{Ker} P$. Then $L_{P}$ is an isomorphism. Define $K_{P}: \operatorname{Im} L \rightarrow \operatorname{Dom} L$ by

$$
K_{P}=L_{P}^{-1}
$$

Then (a) $K_{P}$ is one-to-one and $P K_{P}=0$; (b)on $\operatorname{Im} L, L K_{P}=I$; and (c)on $\operatorname{Dom} L, K_{P} L=$ $I-P$.

Let $N: X \rightarrow Z$ be a continuous mapping. $N$ is called $L$-compact on $\bar{\Omega}$ if $Q N(\bar{\Omega})$ is bounded and $K_{P}(I-Q) N: \bar{\Omega} \rightarrow X$ is compact. Since $\operatorname{Im} Q$ is isomorphic to $\operatorname{Ker} L$, there is an isomorphism

$$
J: \operatorname{Im} Q \rightarrow \operatorname{Ker} L
$$

In the proof of our main theorem, we will use the following result from Gaines and Mawhin [9, page 40].

Theorem 2.1. Let $X$ and $Z$ be two Banach spaces and let $L$ be a Fredholm mapping of index zero. Assume that $N: \bar{\Omega} \rightarrow Z$ is L-compact on $\bar{\Omega}$ with $\Omega$ open bounded in X. Furthermore, assume that

(a) for each $\lambda \in(0,1), x \in \partial \Omega \cap \operatorname{Dom} L$,

$$
L x \neq \lambda N x
$$

(b) for each $x \in \partial \Omega \cap \operatorname{Ker} L$,

$$
\begin{gathered}
Q N x \neq 0, \\
\operatorname{deg}\{J Q N x, \Omega \cap \operatorname{Ker} L, 0\} \neq 0, \quad J Q N: \operatorname{Ker} L \longrightarrow \operatorname{Ker} L .
\end{gathered}
$$

Then the equation $L x=N x$ has at least one solution in $\bar{\Omega} \cap \operatorname{Dom} L$.

We are now in a position to state two lemmas that guarantee our main result. 
Lemma $2.2[6]$. Let $g: \mathbb{Z} \rightarrow \mathbb{R}$ be a function satisfying $g(k+\omega)=g(k), k \in \mathbb{Z}$. Then for any fixed $k_{1}, k_{2} \in I_{\omega}$ and $k \in \mathbb{Z}$,

$$
\begin{aligned}
& g(k) \leq g\left(k_{1}\right)+\sum_{k=0}^{\omega-1}|g(k+1)-g(k)|, \\
& g(k) \geq g\left(k_{2}\right)-\sum_{k=0}^{\omega-1}|g(k+1)-g(k)| .
\end{aligned}
$$

Lemma 2.3. Assume that the following conditions hold:

(H1) $2 m \bar{b}_{1}>\bar{\alpha}_{1}$,

(H2) $\bar{\alpha}_{2}>\bar{b}_{2}$.

Then the system of algebraic equations

$$
\begin{gathered}
\bar{b}_{1}-\bar{a}_{1} u_{1}-\bar{\alpha}_{1} \frac{u_{1} u_{2}}{u_{1}^{2}+m^{2} u_{2}^{2}}=0, \\
\bar{b}_{2}-\bar{\alpha}_{2} \frac{u_{1}^{2}}{u_{1}^{2}+m^{2} u_{2}^{2}}=0
\end{gathered}
$$

has a unique solution $\left(u_{1}^{*}, u_{2}^{*}\right)^{T} \in \mathbb{R}^{2}$ with $u_{i}^{*}>0, i=1,2$.

Proof. Let $v=u_{2} / u_{1}>0$, then from the second equation of (2.14) and in view of (H2), we can solve that

$$
v=\sqrt{\frac{\bar{\alpha}_{2}-\bar{b}_{2}}{m^{2} \bar{b}_{2}}} .
$$

The first equation of (2.14) and (H1) imply

$$
\begin{gathered}
u_{1}^{*}=\frac{\bar{b}_{1}}{\bar{a}_{1}}-\frac{\bar{\alpha}_{1} v}{\bar{a}_{1}\left(1+m^{2} v^{2}\right)} \geq \frac{\bar{b}_{1}}{\bar{a}_{1}}-\frac{\bar{\alpha}_{1} v}{2 m \bar{a}_{1} v}=\frac{1}{2 m \bar{a}_{1}}\left(2 m \bar{b}_{1}-\bar{\alpha}_{1}\right)>0, \\
u_{2}^{*}=v u_{1}^{*}>0 .
\end{gathered}
$$

It follows that the algebraic equations (2.14) have a unique solution $\left(u_{1}^{*}, u_{2}^{*}\right)^{T} \in \mathbb{R}^{2}$ with $u_{i}^{*}>0, i=1,2$. The proof is complete.

Define

$$
l_{2}=\left\{y=y(k): y(k) \in \mathbb{R}^{2}, k \in \mathbb{Z}\right\}
$$

For $\theta=\left(\theta_{1}, \theta_{2}\right)^{T} \in \mathbb{R}^{2}$, define $|\theta|=\max \left\{\theta_{1}, \theta_{2}\right\}$. Let $l^{\omega} \subset l_{2}$ denote the subspace of all $\omega$-periodic sequences equipped with the norm

$$
\|y\|=\max _{k \in I_{\omega}}|y(k)|,
$$


that is,

$$
l^{\omega}=\left\{y=y(k): y(k+\omega)=y(k), y(k) \in \mathbb{R}^{2}, k \in \mathbb{Z}\right\} .
$$

It is not difficult to show that $l^{\omega}$ is a finite-dimensional Banach space.

Set

$$
\begin{aligned}
& l_{0}^{\omega}=\left\{y=y(k) \in l^{\omega}: \sum_{k=0}^{\omega-1} y(k)=0\right\}, \\
& l_{c}^{\omega}=\left\{y=y(k) \in l^{\omega}: y(k)=h \in \mathbb{R}^{2}, k \in \mathbb{Z}\right\} .
\end{aligned}
$$

Then it follows that $l_{0}^{\omega}$ and $l_{c}^{\omega}$ are both closed linear subspaces of $l^{\omega}$ and

$$
l^{\omega}=l_{0}^{\omega} \oplus l_{c}^{\omega}, \quad \operatorname{dim} l_{c}^{\omega}=2 .
$$

Now we state our main result of this section.

Theorem 2.4. Assume that (H1) and (H2) hold. Then (2.4) has at least one positive $\omega$ periodic solution.

Proof. Make the change of variables

$$
N_{1}(t)=\exp \left\{x_{1}(t)\right\}, \quad N_{2}(t)=\exp \left\{x_{2}(t)\right\}
$$

then (2.4) can be reformulated as

$$
\begin{aligned}
& x_{1}(k+1)-x_{1}(k)=b_{1}(k)-a_{1}(k) \exp \left\{x_{1}\left(k-\left[\tau_{1}\right]\right)\right\}-\frac{\alpha_{1}(k) \exp \left\{x_{1}(k)+x_{2}(k)\right\}}{\exp \left\{2 x_{1}(k)\right\}+m^{2} \exp \left\{2 x_{2}(k)\right\}}, \\
& x_{2}(k+1)-x_{2}(k)=-b_{2}(k)+\frac{\alpha_{2}(k) \exp \left\{2 x_{1}\left(k-\left[\tau_{2}\right]\right)\right\}}{\exp \left\{2 x_{1}\left(k-\left[\tau_{2}\right]\right)\right\}+m^{2} \exp \left\{2 x_{2}\left(k-\left[\tau_{2}\right]\right)\right\}} .
\end{aligned}
$$

Define

$$
\begin{aligned}
& X=Y=l^{\omega}, \quad(L x)(k)=x(k+1)-x(k), \\
& (N x)(k)=\left[\begin{array}{c}
b_{1}(k)-a_{1}(k) \exp \left\{x_{1}\left(k-\left[\tau_{1}\right]\right)\right\}-\frac{\alpha_{1}(k) \exp \left\{x_{1}(k)+x_{2}(k)\right\}}{\exp \left\{2 x_{1}(k)\right\}+m^{2} \exp \left\{2 x_{2}(k)\right\}} \\
-b_{2}(k)+\frac{\alpha_{2}(k) \exp \left\{2 x_{1}\left(k-\left[\tau_{2}\right]\right)\right\}}{\exp \left\{2 x_{1}\left(k-\left[\tau_{2}\right]\right)\right\}+m^{2} \exp \left\{2 x_{2}\left(k-\left[\tau_{2}\right]\right)\right\}}
\end{array}\right] \\
& \equiv\left[\begin{array}{c}
\triangle_{1}(k) \\
\triangle_{2}(k)
\end{array}\right] \text {, }
\end{aligned}
$$

for any $x \in X$ and $k \in \mathbb{Z}$. It is easy to see that $L$ is a bounded linear operator,

$$
\operatorname{Ker} L=l_{c}^{\omega}, \quad \operatorname{Im} L=l_{0}^{\omega}, \quad \operatorname{dim} \operatorname{Ker} L=2=\operatorname{codim} \operatorname{Im} L,
$$

then it follows that $L$ is a Fredholm mapping of index zero. 
Set

$$
P x=\frac{1}{\omega} \sum_{k=0}^{\omega-1} x(s), \quad x \in X, \quad Q z=\frac{1}{\omega} \sum_{k=0}^{\omega-1} z(s), \quad z \in Y,
$$

and $P, Q$ are continuous projectors such that

$$
\operatorname{Im} P=\operatorname{Ker} L, \quad \operatorname{Ker} Q=\operatorname{Im} L=\operatorname{Im}(I-Q) .
$$

Furthermore, the generalized inverse to $L$

$$
K_{P}: \operatorname{Im} L \longrightarrow \operatorname{Ker} P \cap \operatorname{Dom} L
$$

exists and can be read as

$$
K_{P}(z)=\sum_{s=0}^{k-1} z(s)-\frac{1}{\omega} \sum_{s=0}^{\omega-1}(\omega-s) z(s)
$$

Thus

$$
\begin{aligned}
Q N x= & {\left[\begin{array}{l}
\frac{1}{\omega} \sum_{k=0}^{\omega-1} \triangle_{1}(k) \\
\frac{1}{\omega} \sum_{k=0}^{\omega-1} \triangle_{2}(k)
\end{array}\right], } \\
K_{P}(I-Q) N x= & {\left[\begin{array}{l}
\frac{1}{\omega} \sum_{s=0}^{\omega-1} \triangle_{1}(s) \\
\frac{1}{\omega} \sum_{s=0}^{\omega-1} \triangle_{2}(s)
\end{array}\right]-\left[\begin{array}{c}
\frac{1}{\omega} \sum_{s=0}^{\omega-1}(\omega-s) \triangle_{1}(s) \\
\frac{1}{\omega} \sum_{s=0}^{\omega-1}(\omega-s) \triangle_{2}(s)
\end{array}\right] } \\
& -\left[\begin{array}{c}
\left(k-\frac{\omega+1}{2}\right) \frac{1}{\omega} \sum_{s=0}^{\omega-1} \triangle_{1}(s) \\
\left(k-\frac{\omega+1}{2}\right) \frac{1}{\omega} \sum_{s=0}^{\omega-1} \triangle_{2}(s)
\end{array}\right] .
\end{aligned}
$$

Obviously, $Q N$ and $K_{P}(I-Q) N$ are continuous. It is not difficult to show that $\overline{K_{P}(I-Q) N(\bar{\Omega})}$ is compact for any open bounded set $\Omega \subset X$ by using the Arzela-Ascoli theorem. Moreover, $Q N(\bar{\Omega})$ is clearly bounded. Thus, $N$ is $L$-compact on $\bar{\Omega}$ with any open bounded set $\Omega \subset X$. 
Now we reach the position to search for an appropriate open bounded set $\Omega$ for the application of the continuation theorem. Corresponding to the operator equation $L x=$ $\lambda N x, \lambda \in(0,1)$, we have

$$
\begin{aligned}
& x_{1}(k+1)-x_{1}(k) \\
& =\lambda\left[b_{1}(k)-a_{1}(k) \exp \left\{x_{1}\left(k-\left[\tau_{1}\right]\right)\right\}-\frac{\alpha_{1}(k) \exp \left\{x_{1}(k)+x_{2}(k)\right\}}{\exp \left\{2 x_{1}(k)\right\}+m^{2} \exp \left\{2 x_{2}(k)\right\}}\right], \\
& x_{2}(k+1)-x_{2}(k) \\
& =\lambda\left[-b_{2}(k)+\frac{\alpha_{2}(k) \exp \left\{2 x_{1}\left(k-\left[\tau_{2}\right]\right)\right\}}{\exp \left\{2 x_{1}\left(k-\left[\tau_{2}\right]\right)\right\}+m^{2} \exp \left\{2 x_{2}\left(k-\left[\tau_{2}\right]\right)\right\}}\right] .
\end{aligned}
$$

Assume that $x(t) \in X$ is an $\omega$-periodic solution of (2.31) for a certain $\lambda \in(0,1)$. Summing on both sides of (2.31) from 0 to $\omega-1$ with respect to $k$, we obtain

$$
\begin{aligned}
& \sum_{k=0}^{\omega-1}\left[x_{1}(k+1)-x_{1}(k)\right] \\
& =\lambda \sum_{k=0}^{\omega-1}\left[b_{1}(k)-a_{1}(k) \exp \left\{x_{1}\left(k-\left[\tau_{1}\right]\right)\right\}-\frac{\alpha_{1}(k) \exp \left\{x_{1}(k)+x_{2}(k)\right\}}{\exp \left\{2 x_{1}(k)\right\}+m^{2} \exp \left\{2 x_{2}(k)\right\}}\right], \\
& \sum_{k=0}^{\omega-1}\left[x_{2}(k+1)-x_{2}(k)\right] \\
& =\lambda \sum_{k=0}^{\omega-1}\left[-b_{2}(k)+\frac{\alpha_{2}(k) \exp \left\{2 x_{1}\left(k-\left[\tau_{2}\right]\right)\right\}}{\exp \left\{2 x_{1}\left(k-\left[\tau_{2}\right]\right)\right\}+m^{2} \exp \left\{2 x_{2}\left(k-\left[\tau_{2}\right]\right)\right\}}\right] .
\end{aligned}
$$

Notice that

$$
\sum_{k=0}^{\omega-1}\left[x_{1}(k+1)-x_{1}(k)\right]=\sum_{k=0}^{\omega-1}\left[x_{2}(k+1)-x_{2}(k)\right]=0 .
$$

Thus

$$
\begin{aligned}
& \bar{b}_{1} \omega=\sum_{k=0}^{\omega-1}\left[a_{1}(k) \exp \left\{x_{1}\left(k-\left[\tau_{1}\right]\right)\right\}+\frac{\alpha_{1}(k) \exp \left\{x_{1}(k)+x_{2}(k)\right\}}{\exp \left\{2 x_{1}(k)\right\}+m^{2} \exp \left\{2 x_{2}(k)\right\}}\right], \\
& \bar{b}_{2} \omega=\sum_{k=0}^{\omega-1} \frac{\alpha_{2}(k) \exp \left\{2 x_{1}\left(k-\left[\tau_{2}\right]\right)\right\}}{\exp \left\{2 x_{1}\left(k-\left[\tau_{2}\right]\right)\right\}+m^{2} \exp \left\{2 x_{2}\left(k-\left[\tau_{2}\right]\right)\right\}} .
\end{aligned}
$$


From (2.31), (2.34), and (2.35), we obtain

$$
\begin{aligned}
& \sum_{k=0}^{\omega-1}\left|x_{1}(k+1)-x_{1}(k)\right| \\
& \quad \leq \sum_{k=0}^{\omega-1}\left[\left|b_{1}(k)\right|+a_{1}(k) \exp \left\{x_{1}\left(k-\left[\tau_{1}\right]\right)\right\}+\frac{\alpha_{1}(k) \exp \left\{x_{1}(k)+x_{2}(k)\right\}}{\exp \left\{2 x_{1}(k)\right\}+m^{2} \exp \left\{2 x_{2}(k)\right\}}\right] \\
& \quad=\left(\bar{B}_{1}+\bar{b}_{1}\right) \omega \\
& \sum_{k=0}^{\omega-1}\left|x_{2}(k+1)-x_{2}(k)\right| \\
& \quad \leq \sum_{k=0}^{\omega-1}\left|b_{2}(k)\right|+\sum_{k=0}^{\omega-1} \frac{\alpha_{2}(k) \exp \left\{2 x_{1}\left(k-\left[\tau_{2}\right]\right)\right\}}{\exp \left\{2 x_{1}\left(k-\left[\tau_{2}\right]\right)\right\}+m^{2} \exp \left\{2 x_{2}\left(k-\left[\tau_{2}\right]\right)\right\}} \\
& \quad=\left(\bar{B}_{2}+\bar{b}_{2}\right) \omega .
\end{aligned}
$$

Note that $x(t)=\left(x_{1}(t), x_{2}(t)\right)^{T} \in X$; then there exist $\xi_{i}, \eta_{i} \in I_{\omega}(i=1,2)$ such that

$$
x_{i}\left(\xi_{i}\right)=\min _{k \in I_{\omega}} x_{i}(k), \quad x_{i}\left(\eta_{i}\right)=\max _{k \in I_{\omega}} x_{i}(k), \quad i=1,2 .
$$

In view of (2.34) and (2.38), we get

$$
\bar{a}_{1} \omega \exp \left\{x_{1}\left(\xi_{1}\right)\right\} \leq \sum_{k=0}^{\omega-1}\left[a_{1}(k) \exp \left\{x_{1}\left(k-\left[\tau_{1}\right]\right)\right\}\right] \leq \bar{b}_{1} \omega
$$

so we get

$$
x_{1}\left(\xi_{1}\right) \leq \ln \left(\frac{\bar{b}_{1}}{\bar{a}_{1}}\right) .
$$

Combining (2.40) with (2.36) and in view of Lemma 2.2, we conclude that

$$
x_{1}(k) \leq x_{1}\left(\xi_{1}\right)+\sum_{k=0}^{\omega-1}\left|x_{1}(s+1)-x_{1}(s)\right| \leq \ln \left(\frac{\bar{b}_{1}}{\bar{a}_{1}}\right)+\left(\bar{B}_{1}+\bar{b}_{1}\right) \omega:=H_{11} .
$$

Also, from (2.34), (2.38), and the inequality $a^{2}+b^{2} \geq 2 a b$, we have

$$
\bar{b}_{1} \omega-\sum_{k=0}^{\omega-1} a_{1}(k) \exp \left\{x_{1}\left(k-\left[\tau_{1}\right]\right)\right\}=\sum_{k=0}^{\omega-1} \frac{\alpha_{1}(k) \exp \left\{x_{1}(k)+x_{2}(k)\right\}}{\exp \left\{2 x_{1}(k)\right\}+m^{2} \exp \left\{2 x_{2}(k)\right\}} \leq \frac{\bar{\alpha}_{1}}{2 m} \omega,
$$

which implies

$$
\bar{a}_{1} \omega \exp \left\{x_{1}\left(\eta_{1}\right)\right\} \geq \bar{b}_{1} \omega-\frac{\bar{\alpha}_{1}}{2 m} \omega
$$


by $(\mathrm{H} 1)$, we get

$$
x_{1}\left(\eta_{1}\right) \geq \ln \left(\frac{2 m \bar{b}_{1}-\bar{\alpha}_{1}}{2 m \bar{a}_{1}}\right)
$$

also, in terms of (2.36) and Lemma 2.2, we obtain

$$
\begin{aligned}
x_{1}(k) & \geq x_{1}\left(\eta_{1}\right)-\sum_{k=0}^{\omega-1}\left|x_{1}(s+1)-x_{1}(s)\right| \\
& \geq \ln \left(\frac{2 m \bar{b}_{1}-\bar{\alpha}_{1}}{2 m \bar{a}_{1}}\right)-\left(\bar{B}_{1}+\bar{b}_{1}\right) \omega=H_{12} .
\end{aligned}
$$

Thus (2.41) and (2.45) lead to

$$
\left|x_{1}(k)\right| \leq \max \left\{\left|H_{11}\right|,\left|H_{12}\right|\right\}:=H_{1} .
$$

On the other hand, from (2.35), we know that

$$
\bar{b}_{2} \omega \leq \sum_{k=0}^{\omega-1} \frac{\alpha_{2}(k) \exp \left\{x_{1}\left(k-\left[\tau_{2}\right]\right)\right\}}{2 m \exp \left\{x_{2}\left(k-\left[\tau_{2}\right]\right)\right\}} \leq \frac{\bar{\alpha}_{2} \omega \exp \left\{H_{11}\right\}}{2 m \exp \left\{x_{2}\left(\xi_{2}\right)\right\}}
$$

which yields

$$
x_{2}\left(\xi_{2}\right) \leq \ln \left(\frac{\bar{\alpha}_{2} \exp \left\{H_{11}\right\}}{2 m \bar{b}_{2}}\right)
$$

in view of (2.37) and Lemma 2.2, we obtain

$$
\begin{aligned}
x_{2}(k) & \leq x_{2}\left(\xi_{2}\right)+\sum_{k=0}^{\omega-1}\left|x_{2}(s+1)-x_{2}(s)\right| \\
& \leq \ln \left(\frac{\bar{\alpha}_{2} \exp \left\{H_{11}\right\}}{2 m \bar{b}_{2}}\right)+\left(\bar{B}_{2}+\bar{b}_{2}\right) \omega=H_{21} .
\end{aligned}
$$

Also, from (2.35) and the monotonicity of the function $x^{2} /\left(x^{2}+a\right)$, we have

$$
\bar{b}_{2} \omega \geq \sum_{k=0}^{\omega-1} \frac{\alpha_{2}(k) \exp \left\{2 H_{12}\right\}}{\exp \left\{2 H_{12}\right\}+m^{2} \exp \left\{2 x_{2}\left(\eta_{2}\right)\right\}} ;
$$

by $(\mathrm{H} 2)$, this leads to

$$
x_{2}\left(\eta_{2}\right) \geq \frac{1}{2} \ln \left(\frac{\bar{\alpha}_{2}-\bar{b}_{2}}{m^{2} \bar{b}_{2}}\right)+H_{12} .
$$


From (2.51), (2.37), and Lemma 2.2, we know that

$$
\begin{aligned}
x_{2}(k) & \geq x_{2}\left(\eta_{2}\right)-\sum_{k=0}^{\omega-1}\left|x_{2}(s+1)-x_{2}(s)\right| \\
& \geq \frac{1}{2} \ln \left(\frac{\bar{\alpha}_{2}-\bar{b}_{2}}{m^{2} \bar{b}_{2}}\right)+H_{12}-\left(\bar{B}_{2}+\bar{b}_{2}\right) \omega:=H_{22} .
\end{aligned}
$$

Combine (2.49) with (2.52), then we can see that

$$
\left|x_{2}(k)\right| \leq \max \left\{\left|H_{21}\right|,\left|H_{22}\right|\right\}:=H_{2}
$$

Obviously, $H_{1}$ and $H_{2}$ are independent of the choice of $\lambda$. By Lemma 2.3, the algebraic equations

$$
\begin{gathered}
\bar{b}_{1}-\bar{a}_{1} u_{1}-\bar{\alpha}_{1} \frac{u_{1} u_{2}}{u_{1}^{2}+m^{2} u_{2}^{2}}=0 \\
\bar{b}_{2}-\bar{\alpha}_{2} \frac{u_{1}^{2}}{u_{1}^{2}+m^{2} u_{2}^{2}}=0
\end{gathered}
$$

have a unique solution $\left(u_{1}^{*}, u_{2}^{*}\right)^{T}$ with $u_{i}^{*}>0(i=1,2)$ under the assumptions in Theorem 2.4.

Let $H=H_{1}+H_{2}+H_{3}$, where $H_{3}>0$ is large enough such that

$$
\left\|\left(\ln \left\{u_{1}^{*}\right\}, \ln \left\{u_{2}^{*}\right\}\right)^{T}\right\|=\max \left\{\left|\ln \left\{u_{1}^{*}\right\}\right|,\left|\ln \left\{u_{2}^{*}\right\}\right|\right\}<H_{3}
$$

and define

$$
\Omega=\left\{x(t)=\left(x_{1}(t), x_{2}(t)\right)^{T} \in X:\|x\|<H\right\} .
$$

It is easy to verify that $\Omega$ satisfies Theorem 2.1(a). When $x \in \partial \Omega \cap \operatorname{Ker} L=\partial \Omega \cap \mathbb{R}^{2}, x$ is a constant vector in $\mathbb{R}^{2}$ with $\|x\|=H$. Then

$$
Q N x=\left[\begin{array}{c}
\bar{b}_{1}-\bar{a}_{1} \exp \left\{x_{1}\right\}-\bar{\alpha}_{1} \frac{\exp \left\{x_{1}+x_{2}\right\}}{\exp \left\{2 x_{1}\right\}+m^{2} \exp \left\{2 x_{2}\right\}} \\
-\bar{b}_{2}+\bar{\alpha}_{2} \frac{\exp \left\{2 x_{1}\right\}}{\exp \left\{2 x_{1}\right\}+m^{2} \exp \left\{2 x_{2}\right\}}
\end{array}\right] \neq 0 .
$$

Since $\operatorname{Im} P=\operatorname{Ker} L, J$ can be chosen as the identity mapping. In view of the assumptions in Theorem 2.4, direct calculation yields

$$
\operatorname{deg}\{J Q N, \Omega \cap \operatorname{Ker} L, 0\} \neq 0 .
$$


By now we have proved that $\Omega$ satisfies all the conditions in Theorem 2.1. Hence (2.23) has at least one solution $\left(x_{1}^{*}(t), x_{2}^{*}(t)\right)^{T}$ in $\operatorname{Dom} L \cap \bar{\Omega}$. Set $N_{1}^{*}(t)=\exp \left\{x_{1}^{*}(t)\right\}, N_{2}^{*}(t)=$ $\exp \left\{x_{2}^{*}(t)\right\}$; then $N^{*}(t)=\left(N_{1}^{*}(t), N_{2}^{*}(t)\right)^{T}$ is a positive $\omega$-periodic solution of (2.4). This completes the proof.

\section{Global asymptotic stability}

The purpose of this section is to present sufficient conditions for the globally asymptotical stability of system (2.4) when the delays are all zero; the method we used here is to construct a suitable Lyapunov function.

Theorem 3.1. Assume that (H1) and (H2) hold and, furthermore, suppose that there exist positive numbers $\nu, c_{1}$, and $c_{2}$ such that

(i)

$$
c_{1} a_{1}(k)+\frac{c_{1} \alpha_{1}(k) N_{2}^{*}(k)\left(m^{2} N_{2}^{* 2}(k)-N_{1}^{* 2}(k)\right)}{\left(N_{1}^{* 2}(k)+m^{2} N_{2}^{* 2}(k)\right)^{2}}-c_{2} \alpha_{2}(k) \frac{2 m^{2} N_{1}^{*}(k) N_{2}^{* 2}(k)}{\left(N_{1}^{* 2}(k)+m^{2} N_{2}^{* 2}(k)\right)^{2}} \geq v,
$$

(ii)

$$
c_{2} \alpha_{2}(k) \frac{2 m^{2} N_{1}^{* 2}(k) N_{2}^{* 2}(k)}{\left(N_{1}^{* 2}(k)+m^{2} N_{2}^{* 2}(k)\right)^{2}}-c_{1} \frac{\alpha_{1}(k) N_{1}^{*}(k)\left(m^{2} N_{2}^{* 2}(k)-N_{1}^{* 2}(k)\right)}{\left(N_{1}^{* 2}(k)+m^{2} N_{2}^{* 2}(k)\right)^{2}} \geq v,
$$

(iii)

$$
a_{1}(k)+\frac{\alpha_{1}(k) N_{2}^{*}(k)\left(m^{2} N_{2}^{* 2}(k)-N_{1}^{* 2}(k)\right)}{\left(N_{1}^{* 2}(k)+m^{2} N_{2}^{* 2}(k)\right)^{2}} \leq 1,
$$

(iv)

$$
\alpha_{2}(k) \frac{2 m^{2} N_{1}^{* 2}(k) N_{2}^{* 2}(k)}{\left(N_{1}^{* 2}(k)+m^{2} N_{2}^{* 2}(k)\right)^{2}} \leq 1
$$

Then the positive solution of system (2.4) is globally asymptotically stable.

Proof. Let $\left\{N_{i}^{*}(k)\right\}(i=1,2)$ be a positive solution of system (2.4). Introduce the change of variables

$$
u_{1}(k)=N_{1}(k)-N_{1}^{*}(k), \quad u_{2}(k)=N_{2}(k)-N_{2}^{*}(k) .
$$


Then, from system (2.4), we can obtain

$$
\begin{aligned}
& u_{1}(k+1) \\
& =N_{1}(k) \exp \left\{b_{1}(k)-a_{1}(k) N_{1}(k)-\frac{\alpha_{1}(k) N_{1}(k) N_{2}(k)}{N_{1}^{2}(k)+m^{2} N_{2}^{2}(k)}\right\} \\
& -N_{1}^{*}(k) \exp \left\{b_{1}(k)-a_{1}(k) N_{1}^{*}(k)-\frac{\alpha_{1}(k) N_{1}^{*}(k) N_{2}^{*}(k)}{N_{1}^{* 2}(k)+m^{2} N_{2}^{* 2}(k)}\right\} \\
& =\left[N_{1}(k) \exp \left\{-a_{1}(k) u_{1}(k)-\alpha_{1}(k)\left[\frac{N_{1}(k) N_{2}(k)}{N_{1}^{2}(k)+m^{2} N_{2}^{2}(k)}-\frac{N_{1}^{*}(k) N_{2}^{*}(k)}{N_{1}^{* 2}(k)+m^{2} N_{2}^{* 2}(k)}\right]\right\}\right. \\
& \left.-N_{1}^{*}(k)\right] \exp \left\{b_{1}(k)-a_{1}(k) N_{1}^{*}(k)-\frac{\alpha_{1}(k) N_{1}^{*}(k) N_{2}^{*}(k)}{N_{1}^{* 2}(k)+m^{2} N_{2}^{* 2}(k)}\right\} \\
& =\left\{\left[1-a_{1}(k) N_{1}^{*}(k)-\frac{\alpha_{1}(k) N_{1}^{*}(k) N_{2}^{*}(k)\left(m^{2} N_{2}^{* 2}(k)-N_{1}^{* 2}(k)\right)}{\left(N_{1}^{* 2}(k)+m^{2} N_{2}^{* 2}(k)\right)^{2}}\right] \frac{u_{1}(k)}{N_{1}^{*}(k)}\right. \\
& \left.+\frac{\alpha_{1}(k) N_{1}^{*}(k)\left(m^{2} N_{2}^{* 2}(k)-N_{1}^{* 2}(k)\right)}{\left(N_{1}^{* 2}(k)+m^{2} N_{2}^{* 2}(k)\right)^{2}} u_{2}(k)+f_{1}\right\} N_{1}^{*}(k+1),
\end{aligned}
$$

$u_{2}(k+1)$

$$
\begin{aligned}
= & N_{2}(k) \exp \left\{-b_{2}(k)+\frac{\alpha_{2}(k) N_{1}^{2}(k)}{N_{1}^{2}(k)+m^{2} N_{2}^{2}(k)}\right\} \\
& -N_{2}^{*}(k) \exp \left\{-b_{2}(k)+\frac{\alpha_{2}(k) N_{1}^{* 2}(k)}{N_{1}^{* 2}(k)+m^{2} N_{2}^{* 2}(k)}\right\} \\
= & {\left[N_{2}(k) \exp \left\{\alpha_{2}(k)\left[\frac{N_{1}^{2}(k)}{N_{1}^{2}(k)+m^{2} N_{2}^{2}(k)}-\frac{N_{1}^{* 2}(k)}{N_{1}^{* 2}(k)+m^{2} N_{2}^{* 2}(k)}\right]\right\}-N_{2}^{*}(k)\right] } \\
& \times \exp \left\{-b_{2}(k)+\frac{\alpha_{2}(k) N_{1}^{* 2}(k)}{N_{1}^{* 2}(k)+m^{2} N_{2}^{* 2}(k)}\right\} \\
= & {\left[\left(1-\alpha_{2}(k) \frac{2 m^{2} N_{1}^{* 2}(k) N_{2}^{* 2}(k)}{\left(N_{1}^{* 2}(k)+m^{2} N_{2}^{* 2}(k)\right)^{2}}\right) \frac{u_{2}(k)}{N_{2}^{*}(k)}\right.} \\
& \left.+\alpha_{2}(k) \frac{2 m^{2} N_{1}^{*}(k) N_{2}^{* 2}(k)}{\left(N_{1}^{* 2}(k)+m^{2} N_{2}^{* 2}(k)\right)^{2}} u_{1}(k)+f_{2}\right] N_{2}^{*}(k+1),
\end{aligned}
$$

where $\left|f_{i}\right| /\|u\|$ converges, uniformly with respect to $k \in \mathbb{N}$, to zero as $\|u\| \rightarrow 0$.

Define a function $V$ by

$$
V(u(k))=c_{1}\left|\frac{u_{1}(k)}{N_{1}^{*}(k)}\right|+c_{2}\left|\frac{u_{2}(k)}{N_{2}^{*}(k)}\right|
$$


where $c_{1}, c_{2}$ are positive constants given in (i). Calculating the difference of $V$ along the solution of the system, we obtain

$$
\begin{aligned}
& \Delta V=c_{1}\left(\left|\frac{u_{1}(k+1)}{N_{1}^{*}(k+1)}\right|-\left|\frac{u_{1}(k)}{N_{1}^{*}(k)}\right|\right)+c_{2}\left(\left|\frac{u_{2}(k+1)}{N_{2}^{*}(k+1)}\right|-\left|\frac{u_{2}(k)}{N_{2}^{*}(k)}\right|\right) \\
& \leq-c_{1}\left\{a_{1}(k)+\frac{\alpha_{1}(k) N_{2}^{*}(k)\left(m^{2} N_{2}^{* 2}(k)-N_{1}^{* 2}(k)\right)}{\left(N_{1}^{* 2}(k)+m^{2} N_{2}^{* 2}(k)\right)^{2}}\right\}\left|u_{1}(k)\right| \\
& +c_{1} \frac{\alpha_{1}(k) N_{1}^{*}(k)\left(m^{2} N_{2}^{* 2}(k)-N_{1}^{* 2}(k)\right)}{\left(N_{1}^{* 2}(k)+m^{2} N_{2}^{* 2}(k)\right)^{2}}\left|u_{2}(k)\right| \\
& -c_{2} \alpha_{2}(k) \frac{2 m^{2} N_{1}^{* 2}(k) N_{2}^{* 2}(k)}{\left(N_{1}^{* 2}(k)+m^{2} N_{2}^{* 2}(k)\right)^{2}}\left|u_{2}(k)\right| \\
& +c_{2} \alpha_{2}(k) \frac{2 m^{2} N_{1}^{*}(k) N_{2}^{* 2}(k)}{\left(N_{1}^{* 2}(k)+m^{2} N_{2}^{* 2}(k)\right)^{2}}\left|u_{1}(k)\right|+\sum_{i=1}^{2} c_{i}\left|f_{i}\right| \\
& =-\left\{c_{1} a_{1}(k)+\frac{c_{1} \alpha_{1}(k) N_{2}^{*}(k)\left(m^{2} N_{2}^{* 2}(k)-N_{1}^{* 2}(k)\right)}{\left(N_{1}^{* 2}(k)+m^{2} N_{2}^{* 2}(k)\right)^{2}}\right. \\
& \left.-c_{2} \alpha_{2}(k) \frac{2 m^{2} N_{1}^{*}(k) N_{2}^{* 2}(k)}{\left(N_{1}^{* 2}(k)+m^{2} N_{2}^{* 2}(k)\right)^{2}}\right\}\left|u_{1}(k)\right| \\
& -\left\{c_{2} \alpha_{2}(k) \frac{2 m^{2} N_{1}^{* 2}(k) N_{2}^{* 2}(k)}{\left(N_{1}^{* 2}(k)+m^{2} N_{2}^{* 2}(k)\right)^{2}}\right. \\
& \left.-c_{1} \frac{\alpha_{1}(k) N_{1}^{*}(k)\left(m^{2} N_{2}^{* 2}(k)-N_{1}^{* 2}(k)\right)}{\left(N_{1}^{* 2}(k)+m^{2} N_{2}^{* 2}(k)\right)^{2}}\right\}\left|u_{2}(k)\right|+\sum_{i=1}^{2} c_{i}\left|f_{i}\right| .
\end{aligned}
$$

Since $\left|f_{i}\right| /\|u\|$ converges uniformly to zero as $\|u\| \rightarrow 0$, it follows from conditions (i) and (ii) that there is a positive $\sigma$ such that if $k$ is sufficiently large and $\|u\|<\sigma$, then

$$
\Delta V \leq-\frac{v}{2}\left\{\left|u_{1}(k)\right|+\left|u_{2}(k)\right|\right\}<-\frac{v}{4}\|u\|
$$

this means that the trivial solution of (3.6) is uniformly asymptotically stable and so is the solution $N^{*}(k)=\left(N_{1}^{*}(k), N_{2}^{*}(k)\right)$ of $(2.4)$.

Notice that

$$
\max \{p(x), q(x)\}=\frac{(|p(x)-q(x)|+p(x)+q(x))}{2} \leq|p(x)|+|q(x)| .
$$

Define

$$
\begin{aligned}
& \Phi(x)=\frac{2 x}{\min \left\{\exp \left\{H_{12}\right\}, \exp \left\{H_{22}\right\}\right\}}, \\
& \Psi(x)=\frac{x}{\max \left\{\exp \left\{H_{11}\right\}, \exp \left\{H_{21}\right\}\right\}} .
\end{aligned}
$$


Then

$$
\Psi(\|u\|) \leq V(u(k)) \leq \Phi(\|u\|)
$$

From the Lyapunov asymptotically stability theorem [1] and in view of the positive definition of $V$ and (3.9), we obtain that the trivial solution of (3.6) is globally asymptotically stable. By the medium of (3.5), we reach the conclusion that the solution $N^{*}(k)=$ $\left(N_{1}^{*}(k), N_{2}^{*}(k)\right)$ of $(2.4)$ is globally asymptotically stable. The proof is complete.

\section{Discussion}

In the previous two sections, we have obtained sufficient conditions for the existence of positive periodic solutions and the globally asymptotical stability of system (2.4) in which the coefficients are periodic. Here we take the response function as the ratio-dependent one: $\varphi(x / y)=c x^{2} /\left(x^{2}+m^{2} y^{2}\right)$. For the system with the corresponding predator response function $\varphi(x)=c x^{2} /\left(1+m x^{2}\right)$, in our previous paper [30], we established verifiable criteria for global existence of positive periodic solutions and stability for the discrete periodic system

$$
\begin{aligned}
& N_{1}(k+1)=N_{1}(k) \exp \left\{b_{1}(k)-a_{1}(k) N_{1}\left(k-\left[\tau_{1}\right]\right)-\frac{\alpha_{1}(k) N_{1}(k) N_{2}(k-[\sigma])}{1+m N_{1}^{2}(k)}\right\}, \\
& N_{2}(k+1)=N_{2}(k) \exp \left\{-b_{2}(k)-a_{2}(k) N_{2}(k)+\frac{\alpha_{2}(k) N_{1}^{2}\left(k-\left[\tau_{2}\right]\right)}{1+m N_{1}^{2}\left(k-\left[\tau_{2}\right]\right)}\right\} .
\end{aligned}
$$

By comparison, we find that the conditions for the existence of positive periodic solutions and the globally asymptotical stability of system (2.4) are weaker than that of system (4.1) when $a_{2}(k)=0$, that is, it is more easier to make the system with ratio-dependent response function have a positive periodic solution than the system with prey-dependent response function. Therefore, the species in a model like (2.4) could live better than those in a model like (4.1). This gives an affirmative exemplum for the claim that the ratiodependent predator-prey theory is more reasonable than the traditional prey-dependent predator-prey theory.

\section{Acknowledgments}

This work was supported by the NNSF of China (10171040), the NSF of Gansu Province of China (ZS011-A25-007-Z), the Foundation for University Key Teacher by the Ministry of Education of China, and the Teaching and Research Award Program for Outstanding Young Teachers in Higher Education Institutions of Ministry of Education of China.

\section{References}

[1] R. P. Agarwal, Difference Equations and Inequalities. Theory, Methods, and Applications, 2nd ed., Monographs and Textbooks in Pure and Applied Mathematics, vol. 228, Marcel Dekker, New York, 2000.

[2] R. Arditi, N. Perrin, and H. Saiah, Functional response and heterogeneities: an experiment test with cladocerans, Oikos 60 (1991), 69-75. 
[3] E. Beretta and Y. Kuang, Global analyses in some delayed ratio-dependent predator-prey systems, Nonlinear Anal. 32 (1998), no. 3, 381-408.

[4] A. A. Berryman, The origins and evolution of predator-prey theory, Ecology 73 (1992), 15301535.

[5] M. Fan and K. Wang, Periodicity in a delayed ratio-dependent predator-prey system, J. Math. Anal. Appl. 262 (2001), no. 1, 179-190.

[6] Periodic solutions of a discrete time nonautonomous ratio-dependent predator-prey system, Math. Comput. Modelling 35 (2002), no. 9-10, 951-961.

[7] Y.-H. Fan, W.-T. Li, and L.-L. Wang, Periodic solutions of delayed ratio-dependent predator-prey models with monotonic or nonmonotonic functional responses, Nonlinear Anal. Real World Appl. 5 (2004), no. 2, 247-263.

[8] H. I. Freedman, Deterministic Mathematical Models in Population Ecology, Monographs and Textbooks in Pure and Applied Mathematics, vol. 57, Marcel Dekker, New York, 1980.

[9] R. E. Gaines and J. L. Mawhin, Coincidence Degree, and Nonlinear Differential Equations, Springer-Verlag, Berlin, 1977.

[10] K. Gopalsamy, Stability and Oscillations in Delay Differential Equations of Population Dynamics, Mathematics and Its Applications, vol. 74, Kluwer Academic, Dordrecht, 1992.

[11] I. Hanski, The functional response of predator: worries about scale, Tree 6 (1991), 141-142.

[12] C. S. Holling, The functional response of predator to prey density and its role in mimicry and population regulation, Mem. Ent. Sec. Can. 45 (1965), 1-60.

[13] S.-B. Hsu, T.-W. Hwang, and Y. Kuang, Global analysis of the Michaelis-Menten-type ratiodependent predator-prey system, J. Math. Biol. 42 (2001), no. 6, 489-506.

[14] - Rich dynamics of a ratio-dependent one-prey two-predators model, J. Math. Biol. 43 (2001), no. 5, 377-396.

[15] H.-F. Huo and W.-T. Li, Periodic solutions of a periodic two-species competition model with delays, Int. J. Appl. Math. 12 (2003), no. 1, 13-21.

[16] H.-F. Huo, W.-T. Li, and S. S. Cheng, Periodic solutions of two-species diffusion models with continuous time delays, Demonstratio Math. 35 (2002), no. 2, 433-446.

[17] V. S. Ivlev, Experimental Ecology of the Feeding of Fishes, Yale University Press, Connecticut, 1961.

[18] C. Jost, O. Arino, and R. Arditi, About deterministic extinction in ratio-dependent predator-prey models, Bull. Math. Biol. 61 (1999), no. 1, 19-32.

[19] Y. Kuang, Delay Differential Equations with Applications in Population Dynamics, Mathematics in Science and Engineering, vol. 191, Academic Press, Massachusetts, 1993.

[20] Y. Kuang and E. Beretta, Global qualitative analysis of a ratio-dependent predator-prey system, J. Math. Biol. 36 (1998), no. 4, 389-406.

[21] W. T. Li, Y. H. Fan, and S. G. Ruan, Periodic solutions in a delayed predator-prey model with nonmonotonic functional response, preprint, 2002.

[22] Y. K. Li, Periodic solutions of a periodic delay predator-prey system, Proc. Amer. Math. Soc. 127 (1999), no. 5, 1331-1335.

[23] Y. K. Li and Y. Kuang, Periodic solutions of periodic delay Lotka-Volterra equations and systems, J. Math. Anal. Appl. 255 (2001), no. 1, 260-280.

[24] R. M. May, Complexity and Stability in Model Ecosystems, Princeton University Press, New Jersey, 1973.

[25] J. D. Murray, Mathematical Biology, Biomathematics, vol. 19, Springer-Verlag, Berlin, 1989.

[26] M. L. Rosenzweig, Paradox of enrichment: destabilization of exploitation ecosystem in ecological time, Science 171 (1971), 385-387.

[27] S. Ruan and D. Xiao, Global analysis in a predator-prey system with nonmonotonic functional response, SIAM J. Appl. Math. 61 (2001), no. 4, 1445-1472. 
[28] Y. Takeuchi, Global Dynamical Properties of Lotka-Volterra Systems, World Scientific Publishing, New Jersey, 1996.

[29] L.-L. Wang and W.-T. Li, Existence of periodic solutions of a delayed predator-prey system with functional response, Int. J. Math. Sci. 1 (2002), no. 1-2, 55-63.

[30] Existence and global stability of positive periodic solutions of a predator-prey system with delays, Appl. Math. Comput. 146 (2003), no. 1, 167-185.

[31] Periodic solutions and permanence for a delayed nonautonomous ratio-dependent predator-prey model with Holling type functional response, J. Comput. Appl. Math. 162 (2004), no. 2, 341-357.

[32] D. Xiao and S. Ruan, Global dynamics of a ratio-dependent predator-prey system, J. Math. Biol. 43 (2001), no. 3, 268-290.

[33] D. Xiao and Z. Zhang, On the uniqueness and nonexistence of limit cycles for predator-prey systems, Nonlinearity 16 (2003), no. 3, 1185-1201.

[34] T. Zhao, Y. Kuang, and H. L. Smith, Global existence of periodic solutions in a class of delayed Gause-type predator-prey systems, Nonlinear Anal. 28 (1997), no. 8, 1373-1394.

[35] H. P. Zhu, S. A. Campbell, and G. S. K. Wolkowicz, Bifurcation analysis of a predator-prey system with nonmonotonic functional response, SIAM J. Appl. Math. 63 (2002), no. 2, 636-682.

Lin-Lin Wang: Department of Mathematics, Tianjing University, Tianjing 300072, China E-mail address: fyh@nwnu.edu.cn

Wan-Tong Li: Department of Mathematics, Lanzhou University, Lanzhou, Gansu 730000, China E-mail address: wtli@lzu.edu.cn 


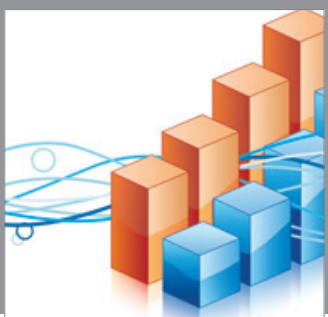

Advances in

Operations Research

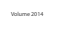

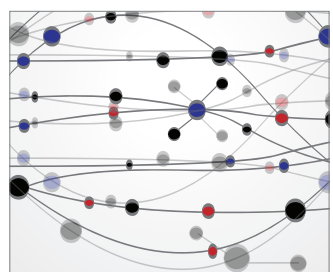

\section{The Scientific} World Journal
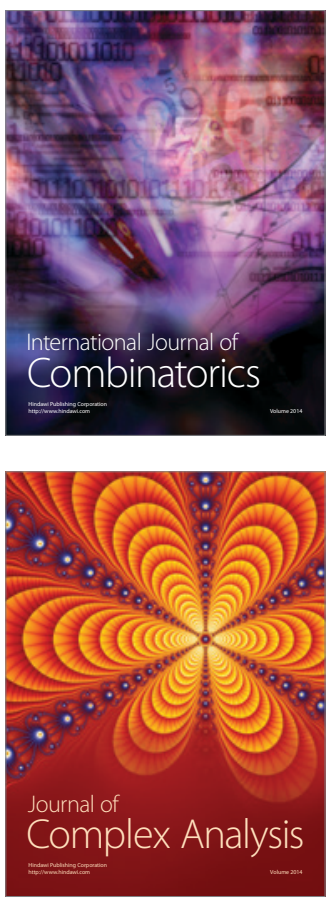

International Journal of

Mathematics and

Mathematical

Sciences
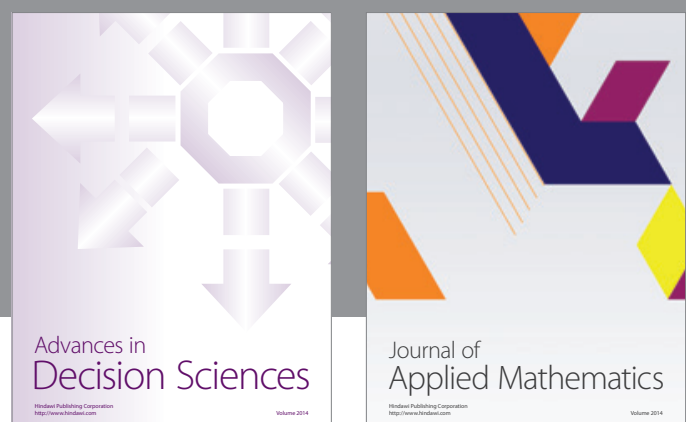

Journal of

Applied Mathematics
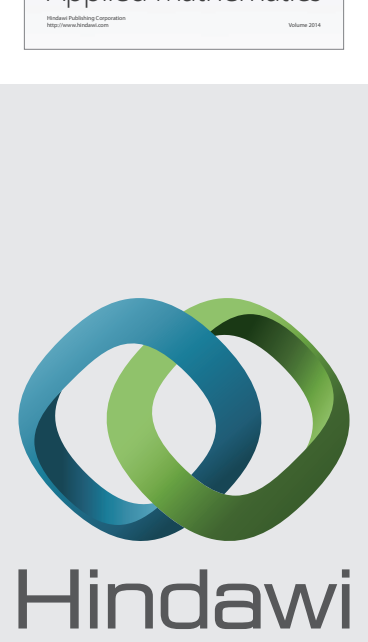

Submit your manuscripts at http://www.hindawi.com
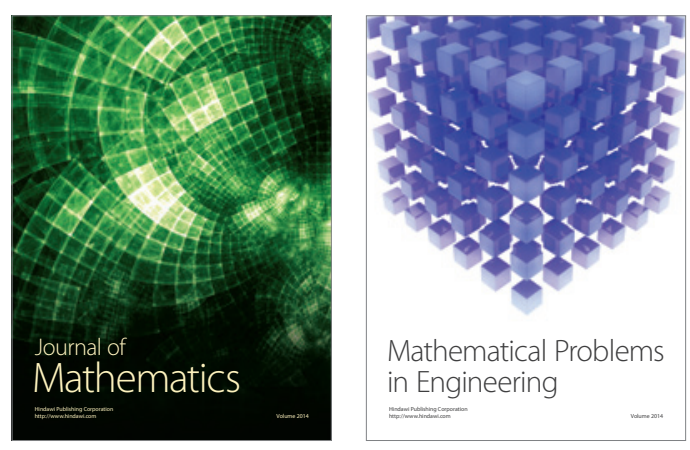

Mathematical Problems in Engineering
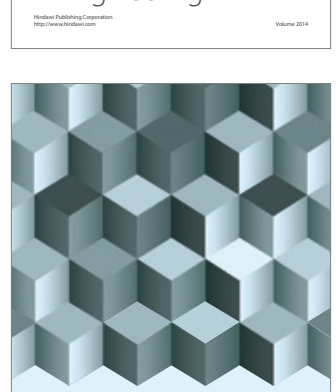

Journal of

Function Spaces
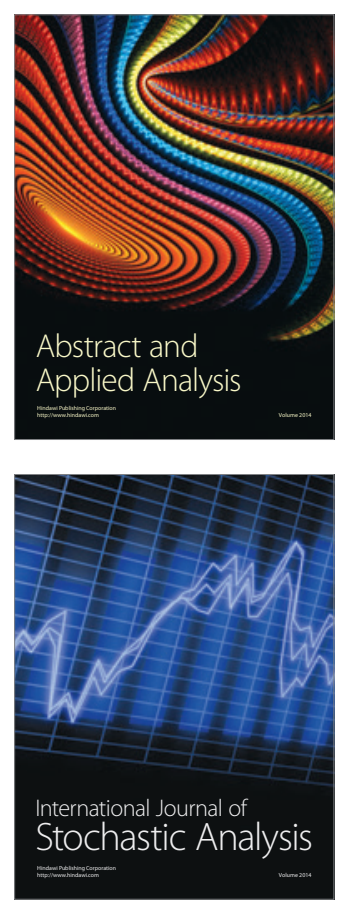

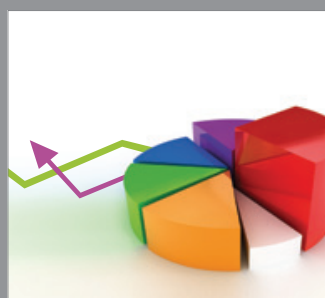

ournal of

Probability and Statistics

Promensencen
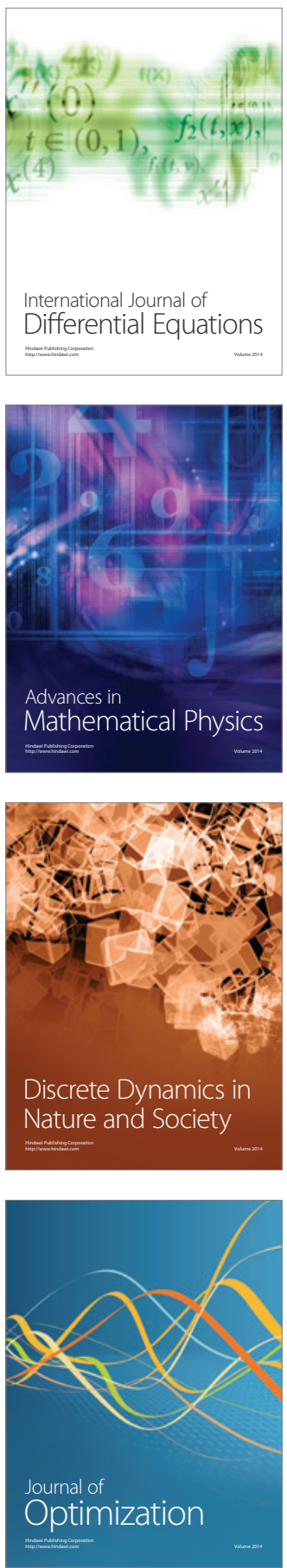